\title{
Addressing the Opioid Epidemic: Recommended Solutions from Physicians
}

\author{
Jessica Cataldo, ${ }_{1}^{1}$ Sandra Collins, ${ }^{1}$ Richard Mckinnies, ${ }^{2}$ Jane Nichols, ${ }^{3}$ Thomas Shaw ${ }^{1}$ \\ ${ }^{1}$ Health Care Management Program, School of Health Sciences; ${ }^{2}$ Radiological Sciences Program, School of Health Sciences; \\ ${ }^{3}$ Rehabilitation Counseling and Administration Program, Rehabilitation Institute, Southern Illinois University, Carbondale, IL, USA
}

\begin{abstract}
The purpose of this study was to assess physicians' accounts related to the current opioid epidemic and to identify solutions that they feel would be most successful in addressing opioid misuse and overuse. A survey was administered a group of physicians obtained from a nationwide database. Nearly all physicians surveyed believed there was a current opioid crisis in the United States and that physicians should take an active role in addressing opioid use in patients. Four key themes emerged regarding solutions to the opioid crisis: i) policy change, ii) improve treatment, iii) education, and iv) alternative treatment. The diversity of responses highlighted the need for a multifaceted approach to address opioid misuse and abuse.
\end{abstract}

\section{Introduction}

In 2017, the Department of Health and Human Services (DHHS $)^{1}$ declared the opioid epidemic a public health emergency. Between 1999 and 2010, the trend of opioid overdoes death rates increased in line with opioid sales. ${ }^{2}$ Furthermore, the percent change in distribution rose as high as $500 \%$ for hydrocodone, $1300 \%$ for methadone, and $1000 \%$ for oxycodone in some states. ${ }^{2}$ In the same period, the age-adjusted mortality rate for opioid-related overdoses nearly quadrupled. ${ }^{3}$

The current epidemic is not the first-time health professionals have searched for ways to combat opioid use disorder. In the mid-to-late nineteenth century, opioid addiction soared as distribution of opium and morphine in-

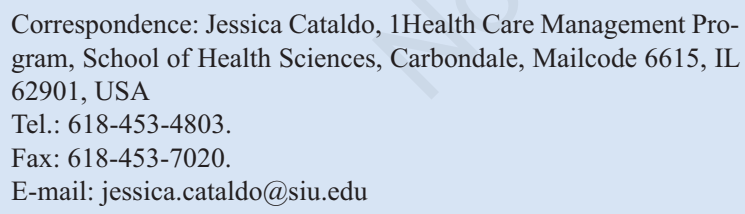

Key words: opioids; opioid misuse; physician perspective.

Conflict of Interest: The Authors declare no potential conflicts of interests.

Received for publication: 11 October 2019.

Accepted for publication: 17 April 2020.

This work is licensed under a Creative Commons Attribution NonCommercial 4.0 License (CC BY-NC 4.0).

${ }^{\circ}$ Copyright: the Author(s), 2020

Licensee PAGEPress, Italy

Qualitative Research in Medicine \& Healthcare 2020; 4:22-30

doi:10.4081/qrmh.2020.8619 creased. ${ }^{4}$ The contributing factors of the epidemic were multifaceted: the introduction of the hypodermic needle, poor understanding of pain and its etiology, and loose regulations on the sale of morphine. Similarly, the approach to address the epidemic was multifaceted: development of alternative pain medication, stricter prescription laws, and prescriber education. ${ }^{4}$

Fast forward to the current opioid epidemic and the discussion looks fairly similar. Contributing factors to the opioid epidemic are multifaceted. King and colleagues ${ }^{5}$ identified key determinants at three levels: prescriber behavior, user behavior and characteristics, and environmental and systemic determinants. Among prescriber behavior, increases in opioid prescriptions, dosages, and prescriptions for methadone and oxycodone were identified as key contributing factors. Among user behavior and characteristics, sociodemographic characteristics and polydrug toxicity were identified as key contributing factors.

There has also been discussion around the role of the fifth vital sign in the current epidemic. In the 1990s, the American Pain Society launched its campaign Pain, The Fifth Vital Sign to increase physician attention to assessment and management of chronic pain in patients which was often overlooked. ${ }^{6}$ The campaign was later adopted by the Joint Commission on the Accreditation of Healthcare Organizations (JCAHO) who mandated pain assessment and treatment in accredited health care facilities. While the intent of the campaign was not to increase the use of opioids to address pain, Campbell ${ }^{7}$ noted that the emphasis on objective pain ratings may have led to an overemphasis on lowering pain ratings with opioids.

In 2017, DHHS launched its 5-Point Strategy to Combat the Opioid Crisis. The strategic framework focuses on improving addiction prevention, treatment, and recovery services; data reporting and collection; pain management care and practice; availability and distribution of over- 
dose-reversing drugs; and research. The framework builds upon previous initiatives and adds new initiatives focused on prevention and treatment. Prescription Monitoring Programs (PMPs) are one example of a previous initiative incorporated into the DHHS' plan. The first PMP dates back to 1918, when New York State required the use of official prescription forms for heroin, cocaine, morphine, opium, and codeine, which were then sent to the health department by the pharmacy. ${ }^{8}$ Today, all 50 states and the District of Columbia have an operational PMP within their borders; however, Missouri does not have a statewide system and 21 states do not have mandatory enrollment for prescribers or pharmacists. ${ }^{9}$

In the present study, we are most interested in the accounts of physicians of the current opioid epidemic and the solutions that they felt would be most successful in addressing the epidemic. Physicians play a key role in addressing the opioid epidemic as key prescribers of opioids. Dunn and colleagues ${ }^{10}$ identified a potential role for physicians in addressing opioid misuse through careful monitoring and oversight of patients when opioids are prescribed. Furthermore, Schnell and Currie ${ }^{11}$ found physicians who completed their initial training at top medical schools prescribed significantly fewer opioids than physicians who completed their training at lower-ranked medical schools. The authors concluded that physician education may be a critical component in addressing the opioid epidemic. Additionally, physicians' attitudes and beliefs toward pain management and opioid use ultimately influence how they practice medicine. ${ }^{12}$ Thus, we found it important to understand the views of physicians in developing a successful plan to address opioids because without their support, efforts are likely fall short. Furthermore, physicians' perspectives provide a complimentary view of addressing the opioid epidemic to the public health approach outlined by DHSS, as medicine tends to be more individualized in its focus. This study allows for comparison in DHSS' strategy to physicians' solutions regarding the opioid epidemic.

A handful of studies have examined physician perspectives around opioid use and misuse, however, these studies tend to focus on either one specialty area or one state. For example, Kennedy-Hendricks and colleagues ${ }^{13}$ found that high-volume opioid prescribers were less likely to support policies aimed to reduce prescription opioid use; however, the study was limited to primary care physicians. Wolfert and colleauges ${ }^{14}$ found that physicians were more likely to view opioids as an acceptable medical practice for chronic cancer pain but less so for pain not related to cancer; however, the study was limited to physicians in Wisconsin. We aim to assess physicians' accounts related to the current opioid epidemic across states and specialty areas to capture multiple perspectives across disciplines or geographic regions and to identify differences that emerge. Additionally, most studies were purely quantitative in focus, limiting responses to predetermined options. We use a mixed method approach to our survey design to elicit more descriptive data from physicians, particularly around potential solutions to the opioid crisis.

\section{Materials and Methods}

\section{Survey Design}

We collected empirical data related to additional solutions physicians felt would be successful in addressing the opioid epidemic via an email survey of physicians across the United States. This data was part of a larger study on accounts of medical marijuana as a replacement for opioids. The total survey instrument included 4 questions related to the current opioid epidemic, 10 questions related to medical marijuana as a replacement for opioids, and 12 demographic questions. The questions related to the current opioid epidemic asked respondents to indicate on a 3-point scale (yes, undecided, no) whether they believed there was a current opioid crisis in the United States and if they believed that physicians should take an active role in addressing opioid usage. Respondents were also asked to indicate their level of agreement on a 5-point Likert scale with four opioid solutions frequently cited in the literature: i) opioid prescribing practices, ii) expanded use and distribution of naloxone, iii) expanded Medication-Assisted Treatment (MAT), and iv) creation of a national prescription database. Lastly, respondents were asked to identify additional solutions they felt would be successful in addressing the opioid epidemic in an openended format. Demographic questions asked respondents to indicate the state they primary practiced in, years they have practiced medicine, whether they were board certified, areas of board certification, size of community, size of practice, professional memberships, hours of direct patient care during a typical week, age, gender, race, and ethnicity.

We developed the survey instrument following a thorough literature review and weekly meetings. Prior to its administration, we tested the survey instrument for clarity, readability, and face validity with a panel of physicians not included in the sampling frame. The survey protocol was approved by the human subjects committee at Southern Illinois University.

\section{Participants and Procedures}

The sampling frame was obtained from a nationwide database company and included a total of 11,939 physicians. We included physicians in family practice, general practice, medical oncology, psychiatry, radiation oncology, pain management, and pain medicine across the United States with all states represented. The survey was sent to physicians via email and was completed using Survey Monkey. The survey was fielded for two weeks. After two weeks, a second survey request was emailed to nonrespondents. 


\section{Data Analysis}

Solutions to the opioid crisis were analyzed using inductive thematic analysis utilizing a realist ontology. ${ }^{15} \mathrm{We}$ aimed to report the accounts and reality of respondents rather than interpret the meaning of responses. Two researchers categorized responses into initial codes independently. Codes were developed based on semantic coding and aimed to summarize the meaning or content of responses. The two coding lists were then compared and utilized to condense codes into key themes through a process of abstraction and clarification. Fifteen initial themes were identified. Through discussions, these fifteen themes were condensed down to four themes plus an other category for responses that did not fit well into the identified themes. The remaining researchers validated the identified themes. Closed-ended survey data were analyzed using SPSS (Version 25, SPSS Inc., USA). Frequency counts and descriptive statistics were calculated for all quantitative survey questions. We included some basic quantitative data for context, however, the focus of the data analysis in this study is on the qualitative data surrounding solutions to the opioid crisis.

\section{Results}

After accounting for undeliverable instruments, a total of 11,483 surveys were delivered successful. The survey yielded 164 total responses for a response rate of approximately $1.4 \%$. As respondents did not always answer every question on the survey, the total participant number (n) varied slightly question to question.

Fifty-five percent of respondents $(n=152)$ were male, with an average age of 57.3 years $(n=141 ; \mathrm{s}=10.9)$. Most respondents were white $(83 \% ; \mathrm{n}=148)$ and non-Hispanic $(93.7 \% ; n=142)$. The four regions of the United States were represented fairly equally in the study. Of the 145 physicians who responded on their location, $46(32 \%)$ were from the Western region, $44(30 \%)$ were from the Midwest region, $26(18 \%)$ were from the Southern region, and $26(18 \%)$ were from the Northeast region. Eightynine percent of respondents $(n=148)$ were board certified, with roughly half of those board certified in family medicine $(n=91)$. Respondents $(n=137)$ practiced medicine for an average of 26.1 years $(\mathrm{s}=10.8)$ and provided an average of 25.7 hours of direct patient care each week $(\mathrm{n}=$ $161 ; \mathrm{s}=17.8)$. Over half of respondents $(\mathrm{n}=145)$ worked in practices with 5 or fewer physicians. Table 1 provides an overview of respondent characteristics.

Eighty-eight percent of respondents $(n=162)$ stated that they felt there was an opioid crisis in the United States, and nearly $99 \%(\mathrm{n}=161)$ believed that physicians should take an active role in addressing opioid use in patients. Respondents indicated high agreement for all four solutions, with nearly $87 \%$ indicating agreement with opioid prescribing practices, $75 \%$ for naloxone, $88 \%$ for ex- panded MAT, and $81 \%$ for a national prescription database. Figure 1 shows the breakdown of responses for each solution.

Eighty-nine total open-ended responses were received regarding additional solutions that respondents felt would be successful in addressing the opioid epidemic. We identified four key themes from these responses: i) policy changes, ii) improve treatment for opioid misuse, iii) education, and iv) alternative treatments for pain. Within each of these key themes, we identified several subthemes.

Table 1. Respondent characteristics.

\begin{tabular}{|c|c|}
\hline $\begin{array}{l}\text { Sex, } \mathrm{n}(\%) \\
\text { rate }(\mathrm{n}) \\
\text { Male, } 90(55.2) \\
\text { Female, } 52(31.9)\end{array}$ & ion response \\
\hline $\begin{array}{l}\text { Age range, years, } \mathrm{n}(\%) \\
30-39,8,(5.7) \\
40-49,26(18.6) \\
50-59,45(32.1) \\
60-69,47(33.6) \\
\geq 70,14(10)\end{array}$ & $87 \%(141)^{*}$ \\
\hline $\begin{array}{l}\text { Race, } \mathrm{n}(\%) \\
\text { White, } 123(83.1) \\
\text { Black, } 3(2) \\
\text { Asian, } 12(8.1) \\
\text { American Indian/Alaskan Native, } 2 \text { (1.4) } \\
\text { Other, } 8 \text { (5.4) }\end{array}$ & $91 \%(148)^{*}$ \\
\hline $\begin{array}{l}\text { Ethnicity, n (\%) } \\
\text { Hispanic, Latino, or Spanish origin, } 9(6.3) \\
\text { Not Hispanic, Latino, or Spanish origin, } 133 \text { (93.7) }\end{array}$ & $87 \%(142)$ \\
\hline $\begin{array}{l}\text { Board certified, n (\%) } \\
\text { Yes, } 131(89.1) \\
\text { No, } 16(10.9) \\
\end{array}$ & $91 \%(148)$ \\
\hline $\begin{array}{l}\text { Area of board certification, } \mathrm{n}(\%) \\
\text { Family Medicine, } 91(54.5) \\
\text { General Practice, } 2(1.2) \\
\text { Internal Medicine, } 5(3.0) \\
\text { Medical Oncology, } 5(3.0) \\
\text { Psychiatry, } 20(12.0) \\
\text { Radiation Oncology, } 1(0.6) \\
\text { Pain Medicine, } 7(4.2) \\
\text { Other, } 36(21.6)\end{array}$ & $90 \%(147)$ \\
\hline $\begin{array}{l}\text { Years of practice, } \mathrm{n}(\%) \\
0-9,9(6.3) \\
10-19,34(23.9) \\
20-29,46(32.4) \\
30-39,40(28.2) \\
\geq 40,13(9.2)\end{array}$ & $87 \%(142)$ \\
\hline $\begin{array}{l}\text { Size of practice, } \mathrm{n}(\%) \\
\text { Solo, } 41(28.5) \\
2-5 \text { physicians, } 44(30.6) \\
6-10 \text { physicians, } 15(10.4) \\
11-30 \text { physicians, } 22(15.3) \\
31-100 \text { physicians, } 10(6.9) \\
\text { More than } 100 \text { physicians, } 12(8.3)\end{array}$ & $89 \%(145)$ \\
\hline
\end{tabular}




\section{Theme 1}

\section{Policy Changes}

Policy changes were the most frequently cited solutions for the opioid crisis, accounting for 22 total responses. However, the types of policy changes recommended spanned four key areas: third-party payers, prescribing limits, criminalization, and prescription monitoring databases.

\section{Third-party payers}

Nine respondents recommended policy changes surrounding third-party payers. Three respondents noted changes in reimbursement toward a value-based model would provide the time necessary to adequately treat pain and addiction issues. One respondent commented: Most important: pay PCPs [primary care physicians] for valuebased care, not time. We can't solve this problem in a 15minute visit. Another respondent suggested significantly reducing reimbursement to pill mills. Four respondents stated increased reimbursement for alternative pain therapies and mental health services for opioid addiction as a key policy change. One respondent recommended mandating third-party payers to cover tamper resistant formulations of opioids, which are designed to make drugs harder to crush, snort, or inject.

\section{Prescribing limits}

Respondents were split on the usefulness of prescribing limits in addressing the opioid epidemic. Four respondents pointed to prescribing limits as a positive solution. One respondent noted: Actually, limiting the ability of physicians to prescribe would greatly help. Or having more specific guidelines on how many to prescribe given the situation, similar to duration of abx [antibiotics] for a given infection. Another respondent stated: Stop prescribing opioids- they have no place in pain management for greater than 1 week. Two respondents felt that prescribing limits would unnecessarily punish responsible patients and providers, and may negatively affect special populations such as those with cancer pain or sickle cell disease. Finally, one respondent did not point to prescribing limits rather to the way in which prescriptions are completed, suggesting, Electronic prescribing of controlled substances with a requirement that each provider have the ability to self-audit prescribing patterns.

\section{Criminalization}

Five respondents believed increased criminalization for drug dealers would help address the opioid epidemic. One respondent suggested a focus on illegal importation, stating: Eliminate and publicize the illegal importation and smuggling of opioids, especially Fentanyl from China. Another respondent felt pharmaceutical companies should be held accountable for creating the current crisis and continuing to profit from it. Similarly, one respondent believed the Drug Enforcement Agency (DEA) should be allowed to investigate drug distribution companies, stating ...much of the 'leaking out' of prescription opiates is from this system gone awry. There are way too many prescription drugs sitting in warehouses ...that is strongly correlated to this problem. A fourth respondent believed those addicted to opioids should be arrested and allowed to dry. However, one respondent felt that the current opioid epi-
Opioid prescribing practices to reduce opioid use disorders and overdose $(n=164)$

Expand use and distribution of naloxone $(\mathrm{n}=163)$

Expand medication-assisted treatment (MAT) to reduce opioid use disorders and overdose $(n=164)$

Create national prescription database $(n=161)$

\section{5}

$6.7 \%$

$6.7 \%$

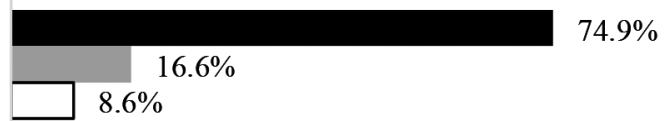

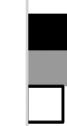

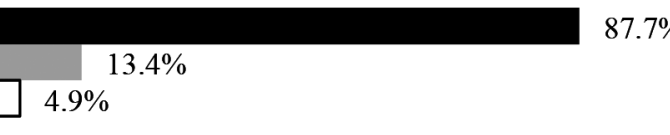

$81.4 \%$
$86.6 \%$

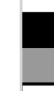

\section{- Agree/Strongly Agree Undecided $\quad \square$ Disagree/Strongly Disagree}

Figure 1. Level of agreement with four opioid epidemic solutions. Percentages may not add to 100 due to rounding. 
demic is partly due to over-criminalization, stating: We need to decriminalize opioid and marijuana use, make these drugs prescription and not scheduled, provide appropriate treatment for patients with abuse disorders and education physicians regarding proper use, just as for any prescription medication.

\section{Prescription monitoring database}

Two respondents recommended mandatory participation in prescription monitoring programs among prescribers. One respondent pointed to the need for a national program, stating: Address the synthetic opiates that are coming across the border. They are too smart for a wall. The other respondent highlighted the need to expand the use of prescription monitoring: Require the $V A$ and Methadone programs to contribute to Rx [prescription] monitoring programs. Have an indication in Rx monitoring programs of ED visits for opioid $O D$ [overdose]/abuse. While not a solution, a third respondent noted a unique challenge associated with prescription monitoring databases, stating: I work in pediatrics and know the parents are "using" but do not have access to their physician drug monitoring program as they are not my patient-identifying a solution here would be helpful.

\section{Theme 2}

\section{Education}

The second most common response related to education for physicians and patients, accounting for 18 total responses. Responses were broken into two categories: physician education and patient education.

\section{Physician Education}

Eleven respondents recommended increased education for opioid prescribers. Recommended length ranged from a minimum of a 3-hour Continuing Medical Education (CME) course to a minimum of an 8-hour CME course. One respondent suggested: Physician education to discourage high-volume open-ended prescribing. Sponsorship of such CME courses was also addressed, noting that pharmaceutical companies should not be allowed to provide CME courses related to opioid prescribing. Medical board intervention for overprescribing providers was also suggested. Two respondents also highlighted a need for physician education on alternative treatments for pain management and dependence disorders.

\section{Patient Education}

Seven respondents noted patient education as a potential solution to the opioid epidemic. Three respondents specifically noted that patients need to be educated that pain cannot be completely eliminated and that some pain should be tolerable. One respondent noted: Health system not focusing on patient satisfaction because they are not satisfied if their pain is not gone; patient education that we cannot eliminate pain completely. Two respondents recommended a national campaign on the dangers of overusing opioids and obtaining opioids on the streets. One respondent recommended early discussions around opioids by introducing education in schools.

\section{Theme 3}

\section{Improve Treatment for Opioid Misuse}

The need for improved treatment to address opioid misuse and abuse was the third most cited solution for the opioid epidemic, accounting for 13 total responses. Respondents addressed three areas for improved treatment: Medication Assisted Treatment (MAT) and other treatment programs, mental health, and screening.

\section{Medication assisted treatment and other treatment programs}

Three respondents specifically identified increased access to MAT as a recommended solution, while five respondents referred generally to treatment programs. One respondent stated: Make MAT as easy as opioids to prescribe. However, another respondent cautioned, MAT with buprenorphine needs to be in monitored format. We don't need Suboxone prescribing which can be quite lucrative to some physicians [and] become the next pill mills. One respondent noted barriers to MAT need to be removed, such as preauthorization requirements or the need for specialty pharmacies. Another respondent highlighted MAT with medications other than buprenorphine, such as Vivitrol and Suboxone, to improve patient functionality and long-term success. Two respondents specifically noted non-MAT treatment as key focuses, including intensive outpatient programs and 12 Step programs. One respondent recommended more funding for opioid addiction treatment programs.

\section{Mental health}

Four respondents noted the importance of mental health care in addressing the opioid epidemic, including counseling and other mental health interventions for those experiencing opioid addiction. The recommended solutions around mental health were generally broad, with two respondents simply stating, Treat mental illness. A third respondent recommended increased reimbursement for wrap-around programs that include mental health treatment and life skills training, while a fourth respondent suggested: Improve access to and affordability of dual diagnosis treatment including group homes.

\section{Screening}

Three respondents highlighted the need for improved screening to determine patients at high risk for opioid addiction. One respondent specifically identified Screening, Brief Intervention, and Referral to Treatment (SBIRT) to identify patients with opioid addiction, while another re- 
spondent noted the need for a standard screening questionnaire and informed consent regarding the risks and disadvantages of opioid use.

\section{Theme 4}

\section{Alternative Treatment}

The fourth most common response related to alternative treatments to address pain, accounting for 12 total responses. Respondents noted three types of alternative treatments for pain: alternative medications to opioids, non-pharmaceutical options, and medical marijuana.

\section{Alternative medications}

Five respondents felt that alternatives to opioids should be focused on to address pain. One respondent felt other options were available from pharmaceutical companies, stating: Find different drugs by pharmaceutical company [that are] not addicting; I am sure pharmaceutical companies [are] hiding solutions. Another respondent noted that buprenorphine was a safer alternative with less risk of dependency and overdose than other opioids. Funding was also highlighted, where one respondent noted: Seriously fund and investigate other modalities for treating chronic pain such as a safer, Cox-2 drug and deep penetrating laser.

\section{Non-pharmaceutical options}

Four respondents highlighted non-pharmaceutical pain treatments as potential solutions to the opioid epidemic, including acupuncture and hypnosis. One respondent noted the need to address all patients holistically in pain management.

\section{Medical marijuana}

Three respondents identified medical marijuana as a potential treatment option in pain management. One respondent suggested: Medical marijuana as [an] adjunctive therapy. Another respondent identified cannabidiol as an alternative treatment option.

\section{Other Responses}

Seven responses did not fit well into the four themes identified. Two respondents did not feel as though any significant measures should be taken to address the opioid epidemic, with one respondent noting: I think public health money should be applied to reduce other preventable causes of death-suicide, guns, and motor vehicles. The "opioid crisis" strikes me as a politcomedia construct; and the other respondent stating: Survival of the fittest; let recreational drug users destroy themselves. No restrictions on terminal patients.

Two respondents felt that physicians need autonomy to treat patients and that government efforts to address the issue are largely ineffective. One respondent noted: Get the government out of medicine. The "pain" fifth vital sign, Joint Commission, state and federal governments have largely created the current issue... Let physicians treat patients as appropriate. Punish the few who violate the law and do not continually control people, physicians [or] patients. Another respondent stated, Opioid abuse is a psychosocial issue. The solutions introduced by federal and state governments are only short-term solutions to this problem. The government has decreased prescriptions of opioids and deaths from heroin, [but] fentanyl ER have increased. This is a MEDICAL problem, not an issue that LAWYERS will be able to solve. We need a completely different approach.

One respondent simply noted that there are no easy solutions to the opioid crisis, while another respondent felt the burden should shift from primary care to pain and mental health specialists. A third respondent noted, Monitor pain management physicians more closely.

\section{Discussion}

While most physicians felt there was an opioid crisis in the United States and that physicians should play an active role in addressing the opioid use, no clear consensus emerged on specific solutions to the opioid epidemic. This may reflect physicians' understanding that the opioid epidemic is multifaceted with several contributing factors and no single solution is likely to be successful alone. It may also reflect physicians' personal experiences treating patients with pain and opioid addiction. In this discussion, we highlight the similarities and dissimilarities between physicians' responses and the DHSS' 5-Point Strategy to Combat Opioid Abuse, Misuse, and Overdose.

Policy changes were most cited with several recommendations made to change reimbursement strategies, including a shift to value-based payment models and increased reimbursement for alternative therapies for pain. While value-based payment models are not explicitly addressed in DHSS' 5-Point Strategy, reimbursement is addressed through the testing of new payment models that incentivize care coordination as well as a waiver demonstration program that allows for Medicaid reimbursement for inpatient and residential addiction treatment. Additionally, payment policies and incentives around the appropriate use of opioids and non-opioid pain treatments are also outlined by DHSS. Shifts to value-based reimbursement, as noted by physicians, aligns with changes occurring throughout the healthcare system. Value-based purchasing may provide a better environment for addressing pain than the traditional fee-for-service model and address concerns raised in the survey regarding inadequate time to treat patient's pain. This will also require physicians to examine their quality metrics around pain and/or opioid use. For performance year 2018, there are nine quality measures related to pain and three quality measures related to opioid use. ${ }^{16}$ 
There has been some movement toward increased access to alternative therapies for pain. The U.S. Pain Federation $^{17}$ noted that several states have passed or introduced legislation to increase assess to alternative or complementary treatment options for patients with chronic pain under state Medicaid programs. Within DHSS' 5-Point Strategy, the veteran pain management research collaborative is supporting greater research related to alternative treatments for pain management, including yoga, massage, and cognitive behavioral therapy.

Roughly $87 \%$ of respondents felt that opioid prescribing practices would reduce opioid use disorders and overdose. In open-ended responses, a few physicians voiced concerns that overly restrictive regulations would negatively affect patients who benefit from the safe use of opioids. DHSS' 5-Point Strategy emphasizes the use of evidence-based guidelines for both opioid and non-opioid treatments, a focus consistent with evidence-based medicine broadly. Several opioid prescribing and treatment guidelines currently exist. ${ }^{18-20}$ Furthermore, Bohnert, Guy, and Losby ${ }^{21}$ found that opioid prescriptions decreased after the release of the CDC's 2016 Guideline for Prescribing Opioids for Chronic Pain. From an applied perspective, healthcare organizations also adopt their own policies regarding opioid prescription practices. Physicians who felt overly restricted may be working in organizations that have adopted stricter policies.

Criminalization is not addressed in DHSS' 5-Point strategy, however, efforts to collaborate with law enforcement, jails, and other community settings are addressed. For example, under the Comprehensive Addiction and Recovery Act, grants were provided to local communities to provide training, medication, and treatment referrals for emergency treatment of opioid overdose. DHSS' strategy focuses more on law enforcement as an intervention point, while responses received around criminalization generally pointed to harsher punishments for opioid abuse. It is important to note that only a handful of respondents felt that criminalization was a potential solution, which may reflect personal beliefs and experiences.

Physician and patient education is included in DHSS' 5-Point Strategy, particularly patient education. This theme appears to be in the greatest alignment between physicians' responses and the 5-Point Strategy. DHSS' plan for patients include science-based public education campaigns around substance use disorders and associated stigma, the use of social and digital media to disperse education, increased school- and community-based prevention programs to prevent opioid misuse, and engagement of faith-based organizations in education efforts. Physician training and education efforts include the development and implementation of a training program in collaboration with professional organizations and education on drug-drug interactions between opioids and other medications. From an applied perspective, ensuring that education efforts are effectively disseminated is particu- larly important. Efforts in developing training programs with professional organizations must be effectively shared with physicians in those professional organizations in a format that is widely adopted and utilized.

Responses received from physicians regarding MAT also aligned closely with efforts outlined in DHSS' 5Point Strategy. MAT is widely discussed in the DHSS' plan, including the creation and dissemination of best practices related to MAT and companion psychosocial treatments. This focus would be beneficial as some physicians voiced concerns around the variability in MAT and the potential for MAT to become problematic itself. Training on evidence-based practices for MAT is also addressed in DHSS' plan to ensure appropriate adoption and implementation of MAT services. Additionally, DHSS' 5Point Strategy addresses barriers to MAT, such innovative services delivery models and working with payers to reduce coverage limitations, a concern specifically noted by one respondent.

Physicians' responses recognized the need for mental health treatment but were not specific in what types of mental health strategies would be most beneficial to address opioid use and misuse. It may be possible that physicians are unsure of mental health strategies that would be most beneficial, which would point to an additional training need for physicians. DHSS' 5-Point Strategy does recognize the need for mental and behavioral health treatment to address the issue, including training for behavioral health providers on substance use disorders and the use of multidisciplinary team models for pain management. Providing physicians with practical approaches to implementing multidisciplinary team models that include mental or behavioral health may be beneficial, particularly for physicians who do not have mental health providers working directly in their practices. Innovative payment models, like those that incentivize care coordination among these teams, would likely increase the adoption of such models.

While only mentioned twice, screening tools to assess potential for risk of opioid dependence may provide physicians with an additional tool in determining the best possible treatment plan for patients with pain issues. Previous research indicates that screening for previous or current illicit drug and alcohol misuse may provide the best indicators. ${ }^{22-24}$ DHSS' 5-Point Strategy includes the development of guidelines for screening on co-occurring mental health and substance use disorders as well as unresolved trauma in people living with chronic pain. Such guidelines would again require effective training and dissemination for widespread adoption. Additionally, physicians, particularly primary care physicians, need to have adequate resources to refer patients who are identified through such screenings. For communities that lack adequate providers and resources for those with substance use disorders, screening efforts will likely feel inadequate, which may be why it was not mentioned frequently by physicians. 
Finally, alternative treatments are addressed in DHSS' plan, however, are somewhat vague in the specific strategies outlined. For example, non-opioid approaches are mentioned several times, which could mean alternative medications to opioids as well as non-pharmaceutical approaches. Based on physician responses, both would be beneficial to better address pain issues among patients. DHSS has invested in the development of non-pharmacologic, non-opioid and/or non-addictive pain therapeutics, as well as novel opioid antagonists to combat synthetic opioids. Such research efforts align with physicians' call for new approaches to pain management. Medical marijuana is not addressed in DHSS' 5-Point Strategy due to its classification as a Schedule I drug under federal law. However, many states have moved forward with allowing the use of medical marijuana for chronic pain patients. The inability to include medical marijuana in data tracking and research efforts in DHSS' plan is a significant gap with efforts being employed at the local level.

\section{Conclusions}

This study provides a snapshot of physician views on the opioid epidemic and its potential solutions. While no clear consensus emerged among physicians on solutions to the current opioid crisis, there were many areas that aligned with the DHSS' 5-Point Strategy to Opioid Use, Misuse, and Abuse. This is promising given the coordinated efforts and significant investment that occurs with the implementation of the DHSS plan. Training and dissemination efforts from DHSS down to local physicians will be key in addressing the concerns noted by physicians on the survey. While many of the solutions noted by physicians are currently included in the DHSS' 5-Point Strategy, it is possible that local physicians are not yet noticing changes of these efforts.

The study involved a relatively small sample size with a low response rate to the overall survey. Additionally, respondents who participated may be those who felt strongly about the opioid epidemic and the use of medical marijuana responded but those with more neutral responses decided not to respond. Although the survey was anonymous, social desirability bias may have led some respondents to answer in a way they perceived to be more socially acceptable. Additionally, respondents did not always answer every question, which increases the issue of item nonresponse in the findings. Lastly, the classification of open-ended responses into key themes was limited to the researchers' understanding of respondents' answers. Using interviews or focus groups to gain better understanding of these responses would strengthen the findings.

Future research may benefit from expanding the survey to a larger group of prescribers, such as nonphysician practitioners and dentists, may provide better insights into potential solutions and their buy-in from the medical com- munity. Very few specialists responded to the initial survey, thus expanding the survey to specific specialists who frequently serve patients with pain may also provide better insights. Both of these steps would allow for a comparison in specific subgroups to identify any potential conflicts or differences. It would also be beneficial to closely examine the implementation of these strategies at an organizational and state level to identify the successful strategies as well as physician pushback. For example, state or organizational policies may dictate specific CME requirements or prescribing limits. Examining the effectiveness of such approaches as well as provider buy-in may provide beneficial insights to curb the opioid trends.

The opioid epidemic is both a public health issue and a medical issue. As such, examining the intersection of the public health response and medical response is necessary to implement a successful, comprehensive response strategy. Our findings show several overlaps between DHSS' 5-Point Strategy and physicians' accounts, which is a positive sign for efforts in curbing the opioid epidemic. There were, however, areas identified by physicians that are not clearly outlined in the 5-Point Strategy, and vice versa, that may point to areas where further alignment and collaboration may benefit the response. Furthermore, understanding physicians' accounts provides insights into the challenges that they face on the front lines of the response and how public health can better support individualized patient care.

\section{References}

1. U.S. Department of Health and Human Services. Strategy to Combat Opioid Abuse, Misuse, and Overdose: A Framework Based on the Five Point Strategy. Available from: https:/www.hhs.gov/opioids/sites/default/files/2018-09/opioid-fivepoint-strategy-20180917-508compliant.pdf. Accessed: October 23, 2018.

2. Jones, CM. Trends in the distribution of selected opioids by state, US, 1999-2011. Presented at the National Meeting of Safe States Alliance; June 5-7, 2013; Baltimore, MD. Available from: https://c.ymcdn.com/sites/safestates.site-ym.com/ resource/resmgr/imported/Jones.pdf. Accessed: October 26, 2018.

3. Chen LH, Hedegaard H, Warner M. Drug-poisoning deaths involving opioid analgesics: United States, 1999-2011. NCHS data brief, 2014, No. 166. Available from: https://www.cdc.gov/nchs/data/databriefs/db166.pdf. September 2014. Accessed: October 26, 2018.

4. Kolodny A, Courtwright DT, Hwang CS, Kreiner P, Eadie JL, Clark TW, Alexander GC. The prescription opioid and heroin crisis: a public health approach to an epidemic of addiction. Annu Rev Public Health 2015;36:559-74.

5. King NB, Fraser V, Boikos C, Richardson R, Harper S. Determinants of increased opioid-related mortality in the United States and Canada, 1990-2013: A systematic review. Am J Public Health 2014;104(8):e32-42.

6. Tompkins DA, Hobelmann JG, Compton P. Providing 
chronic pain management in the "Fifth Vital Sign" era: historical and treatment perspectives on a modern-day medical dilemma. Drug Alcohol Depend 2017;173:S11-21.

7. Campbell JN. The fifth vital sign revisited. Pain 2016;157:3-4.

8. Prescription Drug Monitoring Program Training and Technical Assistance Center. Technical Assistance Guide: History of Prescription Drug Monitoring Programs. Available from: http://www.pdmpassist.org/pdf/PDMP_admin/TAG_History_PDMPs_final_20180314.pdf. Published: March 14, 2018. Accessed: October 27, 2018.

9. Weiner J, Bao Y, Meisel Z. Prescription drug monitoring programs: evolution and evidence. Penn LDI and CHERISH 2017. Available from: http://www.cherishresearch.org/wpcontent/uploads/2017/06/LDI-CHERISH-Brief_PrescriptionDrug-Monitoring-Programs.pdf. Accessed: October 25, 2018.

10. Dunn KM, Saunders KW, Rutler CM, et al. Opioid prescriptions for chronic pain and overdose: a cohort study. Ann Intern Med 2010;152:85-92.

11. Schnell M, Currie J. Addressing the opioid epidemic: is there a role for physician education? Am J Health Econ 2018;4:383-410.

12. Nwokeji ED, Rascati KL, Brown CM, Eisenberg A. Influences of attitudes on family physicians' willingness to prescribe long-acting opioid analgesics for patients with chronic nonmalignant pain. Clin Ther 2007;29:2589-602.

13. Kennedy-Hendricks A, Busch SH, McGinty EE, et al. Primary care physicians' perspectives on the prescription opioid epidemic. Drug Alcohol Depen 2016;165:61-70.

14. Wolfert MZ, Gilson AM, Dahl JL, Cleary JF. Opioid analgesics for pain control: Wisconsin physicians' knowledge, beliefs, attitudes, and prescribing practices. Pain Med 2010;11:425-34.

15. Terry G, Hayfield N, Clarke V, Braun V. Thematic analysis. In: Willig C, Rogers WS, eds. The SAGE handbook of qualitative research in psychology. 2nd ed. Thousand Oaks: SAGE; 2017. p. 17-37.

16. Centers for Medicaid and Medicare Services. Quality Payment Program: Explore Measures. Available from: https:/qpp.cms.gov/mips/explore-measures/quality-measures. Accessed: November 3, 2018.

17. U.S. Pain Foundation. States beginning to support reimbursement for alternative care. Available from: https://uspainfoundation.org/news/states-beginning-to-support-reimb ursement-for-alternative-care/. Published: August 1, 2018. Accessed: March 2, 2020.

18. Centers for Disease Control and Prevention, National Center for Injury, Prevention and Control. CDC Guideline for Prescribing Opioids for Chronic Pain. Available from: https://www.cdc.gov/drugoverdose/prescribing/guideline.ht ml. Revised: August 29, 2017. Accessed: November 5, 2017.

19. Illinois Health and Hospital Association. Opioid Prescribing Guidelines for Patients in the Emergency Department and Immediate Care Centers. Available from: https://www.teamiha.org/files/non-gated/quality/opioid-guidelines-longversion.aspx. Published: April 2018.

20. Overton HN, Hanna MN, Bruhn WE, et al., Opioid After Surgery Workgroup. Opioid-prescribing guidelines for common surgical procedures: an expert panel consensus. J Am Coll Surg 2018;227:411-18.

21. Bohnert ASB, Guy GP, Losby, JL. Opioid prescribing in the United States before and after the Centers for Disease Control and Prevention's 2016 opioid guideline. Ann Intern Med 2018;169:367-75.

22. Ventola CL. Current issues regarding complementary and alternative medicine (CAM) in the United States. Part 1: the widespread use of CAM and the need for better-informed health care professionals to provide patient counseling. P T 2010;35:461-8.

23. Turk DC, Swanson KS, Gatchel RJ. Predicting opioid misuse by chronic pain patients: a systematic review and literature synthesis. Clin J Pain 2008;24:497-508.

24. Compton P, Darakjian J, Miotto K. Screening for addition in patients with chronic pain and "problematic" substance use: evaluation of a pilot assessment tool. J Pain Symptom Manage 1998;16:355-63. 